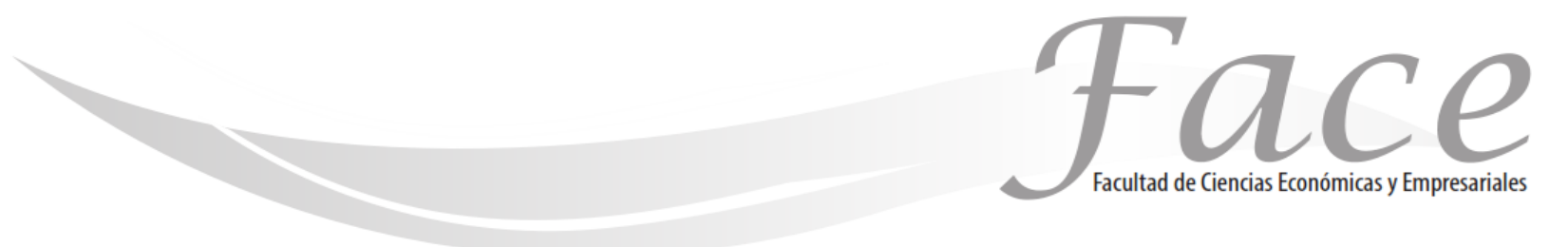

ISSN Impreso: 1794-9920

ISSN Electrónico: 2500-9338

Volumen $19-\mathrm{N}^{\circ} 2$

Año 2019

Págs. $78-89$

(c) (i) $\Theta \Theta$

\title{
SÍNDROME DE BURNOUT EN PROFESIONALES DE SALUD DEL SERVICIO DE URGENCIAS DEL HOSPITAL UNIVERSITARIO DE SANTANDER (ESEHUS)
}

\author{
Alexander Almeida Espinosa * \\ ORCID: https://orcid.org/0000-0002-3030-6831 \\ Dagoberto Torres-Flórez ** \\ ORCID: https://orcid.org/0000-0002-7925-3005 \\ Diego Astudillo Valverde *** \\ ORCID: https://orcid.org/0000-0003-3064-9380
}

Fecha de Recepción: Agosto 7 de 2019

Fecha de Aprobación: Noviembre 8 de 2019

\section{Resumen:}

El síndrome de Burnout se considera uno de los cuadros clínicos principales de aparición medible en el personal de salud, y más en aquel recurso humano, que desarrolla actividades de orden asistencial y están en contacto directo con situaciones propias desencadenantes de factores de estrés, como es el servicio de urgencias en una institución prestador a de servicios de salud. El transcurso básico de la enfermedad, genera episodios incapacitantes medibles, que logran alterar de forma drástica, el funcionamiento normal del servicio de urgencias en el hospital universitario de Santander (Ese Hus). Analizar el impacto administrativo que genera la aparición del síndrome de burnout en los profesionales del servicio de urgencias de la Ese Hus. Estudio de corte transversal con carácter mixto descriptivo y exploratorio, que busca conocer el impacto administrativo de la aparición del síndrome de burnout en profesionales de salud del servicio de urgencias de la Ese Hus. La aparición del síndrome de burnout y su periodo de recuperación, generar impactos negativos administrativos, los cuales van desde el aumento de gastos de personal, desestabilidad laboral, episodios de salud mental identificados, ausencia de sentido de pertenencia y posiblemente, alteración de la calidad en la prestación de servicios de salud en un área de intervención definida de actuación.

Palabras clave: Síndrome de Burnout, estrés laboral, profesionales de salud, instituciones de salud, impactos administrativos

\footnotetext{
* Docente investigador-PhD Ciencias de Salud - PhD en Ciencias Económicas y Administrativas- Colombia. Contacto: alexanderalmeida@10mail.com.

** Docente Universidad Unillanos - Doctorando en Ciencias Económicas y Administrativas. Colombia -Contacto: dtorres@unillanos.edu.co.

*** Docente UniAgustiniana - Magister en Educación. Colombia - Contacto: diegoastudillo@uniagustiniana.edu.co.
} 


\title{
BURNOUT SYNDROME IN HEALTH PROFESSIONALS OF THE EMERGENCY SERVICE OF THE SANTANDER UNIVERSITY HOSPITAL (ESEHUS)
}

\begin{abstract}
:
The Burnout syndrome is considered one of the main clinical tables of onset measurable in health personnel, and more in that human resource, which develops care-order activities and are in direct contact with their own situations Triggers of stress factors, such as the emergency service in an institution providing health services. The basic course of the disease generates measurable disabling episodes that drastically alter the normal functioning of the emergency department at the University Hospital of Santander (Ese Hus). To analyze the administrative impact that the emergence of Burnout syndrome generates in the professionals of the emergency department of the Ese Hus. Cross-sectional study with a descriptive and exploratory mixed character, which seeks to know the administrative impact of the onset of Burnout syndrome in health professionals of the emergency service of Ese Hus. The onset of Burnout syndrome and its recovery period, generate negative administrative impacts, ranging from increased personnel expenses, labor destability, identified mental health episodes, absence of sense of belonging and Possibly, alteration of the quality in the provision of health services in an area of defined intervention of action.
\end{abstract}

Keywords: Burnout syndrome, work stress, health professionals, health institutions, administrative impacts

\section{SÍNDROME DA QUEIMADURA EM PROFISSIONAIS DE SAÚDE DO SERVIÇO DE EMERGÊNCIA DO HOSPITAL DA UNIVERSIDADE DE SANTANDER (ESEHUS)}

\author{
Resumo:
}

A síndrome de Burnout é considerada uma das principais tabelas clínicas de início mensuráveis no pessoal de saúde e mais nesse recurso humano, que desenvolve atividades de assistência e está em contato direto com suas próprias situações. Gatilhos de fatores de estresse, como o serviço de emergência em uma instituição prestadora de serviços de saúde. 0 curso básico da doença gera episódios mensuráveis incapacitantes que alteram drasticamente o funcionamento normal do departamento de emergência do Hospital Universitário de Santander (Ese Hus). Analisar o impacto administrativo que o surgimento da síndrome de Burnout gera nos profissionais do pronto socorro de Ese Hus. Estudo transversal, de caráter descritivo e exploratório misto, que busca conhecer o impacto administrativo do aparecimento da síndrome de Burnout nos profissionais de saúde do serviço de emergência de Ese Hus. $O$ início da síndrome de Burnout e seu período de recuperação geram impactos administrativos negativos, que vão desde aumento de gastos com pessoal, desestabilização do trabalho, episódios de saúde mental identificados, ausência de sentimento de pertença e, possivelmente, alteração da qualidade na prestação de serviços de saúde em uma área de intervenção definida de ação.

Palavras-chave: Síndrome de Burnout, estresse no trabalho, profissionais de saúde, instituições de saúde, impactos administrativos 


\section{INTRODUCCIÓN:}

El síndrome de Burnout, es considerado como uno de los cuadros clínicos de aparición más frecuentes y que logran afectar de forma trascendental la salud de los individuos, así como su rendimiento profesional (Hamson, 2015). El personal asistencial de salud, que labora en las instituciones de salud no escapa a esta condición, la que repercute en la calidad de los servicios que ofrece y en la satisfacción propia del trabajo ejercido.

De igual forma, se considera como un síndrome clínico de preocupación, por las repercusiones personales que implica su aparición, ya sean emocionales, familiares conductuales o psicosomáticas y sociales. A su vez, se suma características propias del ámbito laboral $u$ organizacional, como ausentismo laboral, disminución del grado de satisfacción de los profesionales y pérdida de productividad (Borda y Navarro, 2010).

Este síndrome conlleva a cambios negativos, entre ellos el cansancio corporal, alteraciones del apetito y sueño, maltrato a las personas con las que interactúan; así como, la falta de realización profesional donde se manifiestan conductas pseudo depresivas y baja productividad en el trabajo, lo que lleva a una escasa 0 nula realización profesional (Hernández, 2012)

Estos episodios de aparición, representan una de las patologías más frecuentes la depresión y cuadros de salud mental (Romero, 2010), atribuida a las vivencias cotidianas en el área laboral de los profesionales y su relación con el entorno de trabajo y los resultados productivos de abordaje clínico - administrativo que deben dar cumplimiento.

Así mismo, estudios encontrados indican que en profesiones de salud como médicos, enfermeras, fisioterapeutas, microbiólogos, entre otros; presentan problemas de salud física y mental (Peiró, 2010), ocasionado por la carga laboral extensa, los turnos asignados de trabajo y la humanización del servicio de salud, que deben dar y estar expuestos a recibir por parte de los usuarios que consultan el servicio y la relación costo - efectiva con los mismos profesionales y auxiliares del área de desempeño.
El síndrome incluye, entre otros aspectos, agotamiento emocional, despersonalización o deshumanización, empoderamiento de actitudes negativas, de insensibilidad y de cinismo hacia los receptores de servicio prestado y falta de realización profesional (Tordera y Mañas, 2011), con tendencias a evaluar el propio trabajo de forma negativa, con vivencias de insuficiencia profesional y baja autoestima personal; adicionalmente se pueden identificar síntomas físicos de estrés, como cansancio y malestar general (Romero, 2010).

Autores como Adali (2013), hacen mención de las consecuencias iniciales del síndrome de burnout a nivel individual, ya que la persona afectada tiene dificultades para concentrarse o para tomar decisiones, se comporta de forma cínica, realiza una autocrítica orientada hacia la desvalorización propia, el autosabotaje, la desconfianza y la baja consideración hacia el propio trabajo.

Las segundas consecuencias que se producen sobre la conducta del trabajador y la organización donde desarrolla su trabajo (Jaurequi, 2012); es así como, la empresa también paga un alto coste por tener trabajadores "quemados" ya que causan retrasos, disminuyen su productividad, aumentan las ratios de absentismo, piden ser cambiados de puesto, disminuyen su rendimiento laboral y el compromiso con la organización, realizan un trabajo de baja calidad, se ven involucrados en incidentes y accidentes, muestran públicamente sus intenciones de abandonar el trabajo, se quejan y reclaman constantemente.

Finalmente, se suma las consecuencias producidas a nivel familiar, ya que se ha observado que las personas con altos niveles de burnout tienen menos satisfacción con la pareja y las conductas en el hogar son de irritación, disgusto y tensión. Esta situación incide especialmente en el deterioro de las interacciones personales y realimenta las consecuencias de los otros niveles. (Alpi, y Alvaran, 2014)

Por lo anterior, se hace necesario analizar el impacto administrativo, que evidencia el síndrome de burnout en los profesionales del servicio de urgencias de la Ese Hus, en el primer semestre del año en curso, con el fin de establecer acciones de mejoramiento, que favorezcan la continuidad operacional de la prestación de servicio, sin afectar la costó - efectividad institucional y la satisfacción del cliente interno y externo institucional. 


\section{SÍNDROME DE BURNOUT}

El Estrés Laboral ha sido un tema ampliamente debatido y analizado en muchas investigaciones debido al efecto que produce sobre la salud de los trabajadores (Pereira, Gálvez y Herrer, 2011) y las condiciones psicológicas y sociales (Hernández, 2012). Las Instituciones dedicadas a la atención de salud, como Hospitales, Centros de Salud, entre otros no se escapan a esta forma de estrés dadas las condiciones de trabajo, las cuales varían en las diferentes entidades de salud, pero que tienen en común el volumen y el manejo del dolor humano.

El concepto de Burnout, se aborda por primera vez en 1974 por Freudenberger, (Beas, 2010), defendiéndolo como "el fallo de sujeto ante el manejo del estrés", planteando que las personas a riesgo tiene una personalidad débil y baja tolerancia al estrés. Es así como sugiere que un "perfil de las personas con riesgo a desarrollar el síndrome se caracterizan por pensamientos muy idealistas, y constancia hacia el trabajo para conseguir metas personales y lograr el bien común, pero a pesar del esfuerzo y compromiso los profesionales fracasan" (Rivera, 2016).

Es por esto, que en esta época las publicaciones hacian más énfasis en las diferencias individuales (personalidad) más que en las condiciones medio ambientales del trabajador. Pero, solo hasta el año de 1977, cuando Maslach, expone sus trabajos en Psicología, en una convención de la Asociación Americana de Psicólogos (APA), se utiliza el término Burnout, formalmente, donde referencia el desgaste profesional de personas que están vinculadas a los sectores de servicios humanos, profesionales de la salud y de la educación bajo condiciones difíciles en contacto directo con los usuarios (Barrios y Sánchez, 2015).

El término de Burnout puede delimitarse desde dos perspectivas, la clínica y la psicosocial (Cortés y García, 2012). La clínica, lo ve como el estado al cual llega una persona que está sometida al estrés laboral (Peñacoba,
2010) y la psicosocial lo plantea como el resultado de las interacciones del medio ambiente y las condiciones personales que hacen que tengan diferentes manifestaciones según los momentos (Moreno, 2011).

Garrosa y González (2010), hacen énfasis que el síndrome de Burnout es una manifestación comportamental del estrés laboral, y lo entienden como un síndrome tridimensional caracterizado por cansancio emocional (CE), despersonalización (DP) en el trato con clientes y usuarios, y dificultad para el logro/realización personal (RP). El síndrome de Burnout ha sido ampliamente discutido y se han desarrollado varias definiciones partiendo de enfoque teóricos variados, es así como los ambientalistas dan una definición sistémica, consideran al estrés como algo externo al sujeto y enfocan al Burnout como respuesta a la interacción entre el individuo y el entorno laboral, permitiendo la utilización de estrategias para intentar el cambio o eliminación de factores predisponentes (Novoa y Sánchez, 2015).

Autores como Chalving (2010), señala cuatro variables importantes para explicar la aparición y el desarrollo del Burnout: 1) la presión de satisfacer las demandas de otros, 2) intensa competitividad, 3) deseo de obtener más dinero y 4) sensación de ser desprovisto de algo que se merece.

Otras posturas lo definen desde un enfoque del individuo, en los ochenta, lo explica como un proceso de adaptación del estrés laboral, que se caracteriza por desorientación profesional, desgaste, sentimientos de culpa por falta de éxito profesional, frialdad o distanciamiento emocional y aislamiento (Macarry, 2013).

Así mismo, Misth (2010), hace énfasis que el contenido del síndrome de burnout tiene que ver con una pérdida de las fuentes de energía del sujeto y lo define como "una combinación de fatiga física, cansancio emocional y cansancio cognitivo"; el cual faceta de forma directa el rendimiento personal y profesional del individuo, en el rol o 
desempeño de sus funciones básicas cotidianas.

Por otro lado, Parraga (2015), no solo plantea la definición del síndrome, sino que establece la relación de los grupos específicos de aparición, siendo desde los perfiles profesionales, hasta las amas de casa, las personas que más sufren el desenlace de este cuadro clínico y a su vez, repercute en el rendimiento funcional de los mismos,

Simado a esto, autores como Tous (2014), mencionan que las personas en su esfuerzo por adaptarse y responder eficazmente a las demandas y presiones laborales, se esfuerzan en exceso de manera prolongada en el tiempo. Añadiendo a esto, la sobre exigencia, la tensión y la frustración por resultados no esperados, se originan riesgos de enfermar física (alteraciones psicosomáticas) y/o mentalmente (estados ansiosos, depresivos, alcoholismo, etc.).

Según Mingote y Santiago (2013), en sus trabajos investigativos, argumentan que este "síndrome puede ocurrir en todos los ámbitos de la acción del sujeto, pero ha sido estudiado en mayor medida en el campo de los profesionales asistenciales y se ha extendido en profesiones diversas"; es así, cuando los trabajadores presentan estos síntomas, hay un deterioro en el rendimiento profesional y en consecuencia, puede derivar a un deterioro en la calidad del servicio, ausentismo laboral, alto número de rotaciones y abandono del puesto de trabajo factores que facilitan el síndrome y quienes son los más vulnerables (Beas, 2010).

Las recientes investigaciones parten de un perspectiva integradora donde no sólo se consideran las variables relacionadas con la organización, sino que también se cuestiona el por qué las personas responden de manera diferente al estrés y el tipo de interacción entre las variables organizacionales y personales (Adali, 2013).

Los estudios epidemiológicos sobre estrés laboral 0 burnout sugieren la necesidad de una mayor consideración sobre los factores psicológicos, ya que los estresores psicológicos vinculados con los aspectos organizacionales (trabajo) y no organizacionales (sucesos vitales y estrés diario), son agentes causales de alteraciones físicas que pueden ser mediados por la evaluación cognoscitiva y las estrategias de afrontamiento, y eventualmente moderados por diferentes factores personales (Borda y Navarro, 2010).

Hernández (2012), plantea como factores predisponentes al Síndrome de Burnout Variables Personales AfectivoCognitivo-Emocionales, en las cuales incluye la indefensión aprendida, ausencia de características de personalidad resistente, demandas emocionales, estrategias de afrontamiento inadecuadas, déficit en autoeficacia, patrón de conducta tipo $A$, neuroticismo ansiedad-rasgo, baja tolerancia a la ambigüedad, locus de control externo, déficit en auto concepto y autoestima, expectativas personales e intereses sociales muy altos; Variables Personales Biológico - Demográficas, en donde él incluye, sexo y edad.

En las Variables del Contexto Ambiental como el apoyo social, comunicación, satisfacción vital y recursos de afrontamiento familiares; la Variable Organizacional, dada por la sobrecarga de trabajo, la ambigüedad del rol, falta de control o autonomía en el trabajo, cultura corporativa e incongruencia organizacional; Variables del Contexto Laboral son inadecuación profesional, adicción al trabajo, vivencia continua de sufrimiento y de la muerte, carga mental del trabajo, ergonómicos, trabajo por turnos y nocturnos y Variables Estructurales como las políticas de la dirección de la empresa.

Con respecto a los factores o variables personales, específicamente las afectiva-cognitiva-emocional, que contribuyen o no a mantener la salud, Kobasa en 1982 construye un modelo teórico desde la Psicología social del estrés y la salud (Guevara y Herrera, 2011), con una perspectiva salutogénica, en el cual plantea el concepto de Personalidad Resistente al Estrés, construido por tres dimensiones fundamentales. 
Mauricio Ortigosa Hernández

La primera es el compromiso, que lo define como la tendencia a implicarse en todas las actividades de vida y a identificarse con el significado del propio trabajo, implica en la persona un reconocimiento de metas, una capacidad de toma de decisiones y mantenimiento de los valores.

La segunda dimensión, el control, definido como la convicción de poder trasformar 0 intervenir en los acontecimientos y finalmente, la última corresponde al reto, definida como la creencia de la capacidad de sí mismo para manejar situaciones de crisis y de cambio por percibir una oportunidad de desarrollo alrededor de estas situaciones. Peiró (2010), corrobora la hipótesis planteada por Kobasa, ya que mostró una reducción del Burnout y mejor salud en profesores quienes puntuaron alto en Personalidad Resistente, implica por lo tanto que las variables personales y fundamentalmente cognitivas $y$ motivaciones influyen en el desarrollo del Burnout.

Por otra parte, el apoyo social y la autonomía en el puesto de trabajo también han sido considerados como importantes antecedentes del burnout. Adicionalmente, las investigaciones sobre el burnout han identificado al entorno físico y las características estresantes del propio puesto de trabajo, el desempeño del rol laboral, las relaciones de trabajo y del desarrollo de la carrera en la organización, las formas organizativas, la propia estructura, el clima organizacional, éste último definido como las percepciones compartidas que tienen los miembros de una organización acerca de los procesos organizacionales, tales como las políticas, el estilo de liderazgo, las relaciones interpersonales y la remuneración que impactan en la satisfacción laboral del empleado (García y Larios-Gómez, 2017). Asimismo, la implantación de nuevas tecnologías, entre otros, como los principales desencadenantes del estrés en el ambiente laboral (Vega, Sanabria y Sánchez, 2012).

El estudio de Salazar y Beaton (2010), analizan la influencia de las variables organizacionales y personales, personalidad resistente y sentido de la coherencia dentro del proceso de génesis y desarrollo del burnout, con el fin de determinar si ambos constructos pueden ser entendidos como variables de carácter salutogénico que actúan como factores protectores que disminuyen la probabilidad de que el individuo desarrolle y experimente burnout (Jaurequi, 2012). Estos resultados muestran definitivamente que las variables de personalidad resistente, sentido de coherencia, apoyo social y autonomía en el trabajo definitivamente disminuyen la probabilidad del desarrollo del Burnout.

El apoyo social moderado hace que se tenga un agotamiento emocional moderado y se desarrolle bajos niveles de deshumanización. El concluye que los factores predictores del burnout, son el no creer en el trabajo, no afrontar el estrés ni aquello que lo provoca; estas actitudes y conductas llevan a un incremento del mismo. Por el contrario los factores protectores son las habilidades adquiridas para enfrentar los estresores, creer en el trabajo y la sensación de compromiso y pertenencia; Éstas últimas variables facilitan que se perciba el estrés como realmente es: sin magnificarlo para no desarrollar fastidio por su trabajo. Así mismo, aumenta la probabilidad de enfrentar sus actividades de una manera adecuada para no llegar al burnout. Finalmente, la evidencia empírica sugiere que el apoyo social puede reducir el estrés laboral. (Mingote y Santiago (2013).

Peñacoba (2010), plantea unos factores protectores a nivel personal y laboral, los personales son los valores personales, el tiempo con la familia y amigos, la actividad religiosa o espiritual, el auto cuidado, la filosofía de vida saludable y el tener un mentor. A nivel laboral, el control de la sobrecarga, encontrar el significado en el trabajo y establecer límites, tener adecuado soporte o apoyo del sistema administrativo y tener personas de soporte y guía. Plantea además unos factores condicionantes a nivel personal y laboral, los personales son la personalidad tipo $\mathrm{A}$, el incumplimiento de las metas personales y la personalidad compulsiva. A nivel laboral, la sobrecarga, el poco soporte social, la pérdida de control y las políticas administrativas.

El síndrome de Burnout tiene una multitud de variables que facilitan su presentación lo cual hace más difícil el manejo 
del mismo en ambientes laborales dedicados al manejo de la salud (Tordera y Mañas, 2011). El Burnout es un problema de salud pública que afecta en forma significativa diferentes grupos ocupacionales y es fundamental comprender realmente las interacciones que se dan, ya que las relaciones son complejas.

\section{MATERIALES Y METODOS}

Estudio de corte transversal con carácter mixto descriptivo y exploratorio, cuya población objetiva, fue de 42 profesionales que laboran en el servicio de urgencias de la Ese Hus (total de población); la población se seleccionó, debido a que el desarrollo de sus funciones obedecen a los dos turnos de 12 horas, en los que se prestan modelos de atención institucional de cuarto nivel de complejidad clínica.

De forma inicial, se construyó una encuesta con preguntas sobre aspectos sociodemográficos y laborales: edad, estado civil, nivel académico, antigüedad laboral, antigüedad de adscripción al hospital, categoría, servicio y turno laboral. Para evaluar el síndrome de burnout se aplicó el cuestionario Maslach Burnout Inventory (MBI). Este cuestionario fue validado por Maslash y Jackson apareciendo su versión definitiva en 1986. Se utilizó una versión traducida y validada de la original que ya ha sido utilizada en numerosos estudios realizados (Aranda, 2010

La escala se compone de un cuestionario de 22 ítems con 7 opciones de respuesta (escala de likert de 0 a 6 ), desde nunca a diariamente. Las puntuaciones de cada escala se obtienen al sumar los valores de los 22 ítems. Como punto de corte no hay un acuerdo general entre los autores, sin embargo en la mayoría se ha utilizado el punto corte dividiendo el puntaje total en tres tercios, siendo el tercio superior para Diagnóstico de Burnout, tercio medio tendencia a burnout y tercio inferior sin riesgo de padecer burnout, con puntaje máximo de 132 y mínimo de 0 .

Esta escala representa una manera objetiva de medir y determinar el nivel de burnout que experimenta una persona, en cuanto a sus tres subescalas: CE, DP y RP. El cuestionario consta de 22 ítems con respuestas en escala de puntuación tipo Likert (0-6), de los cuales 9 valoran (CE) Cansancio Emocional, 5 la (DP) Despersonalización y 8 la (RP) Realización Personal
Es así como en la subescala de agotamiento o cansancio emocional. Valora la vivencia de estar exhausto emocionalmente por las demandas del trabajo. Consta de 9 preguntas $(1,2,3,6,8,13,14,16,20$.) Puntuación máxima 54. 2. En la Subescala de despersonalización. Valora el grado en que cada uno reconoce actitudes frialdad y distanciamiento Está formada por 5 ítems $(5,10,11,15$, 22.) Puntuación máxima 30; finalmente, en la subescala de realización personal. Evalúa los sentimientos de autoeficiencia y realización personal en el trabajo. Se compone de 8 ítems $(4,7,9,12,17,18,19,21$. Puntuación máxima 48.

\section{Criterios de inclusión}

- Profesionales de salud que labora en el servicio de urgencias de la institución

\section{Criterios de exclusión}

- Personal de salud en proceso de formación académica (estudiantes y residentes).

- Profesionales de salud que diligenciaron de manera errónea e incompleta el cuestionario.

- Profesionales de salud encontraban en periodo de vacaciones o periodo de licencia.

\section{Consideraciones Éticas}

De acuerdo a la Resolución 8430 de 1993 de la normatividad colombiana y a la declaración de Helsinki, en este estudio se considera de riesgo ético mínimo teniendo en cuenta que algunas preguntas incluidas en el cuestionario pudieron generar algún grado de susceptibilidad dado que se tocaron temas relevantes que influyen en la calidad de vida de las personas. Se aplicó un formato de consentimiento informado previo a la obtención de la información y se realizó una recolección de datos con cuestionarios anónimos y sin diligenciar la entidad a la cual se pertenecía con el fin de mantener reserva absoluta de la misma. Dentro del consentimiento informado se incluyó una nota aclaratoria sobre el deseo de los participantes de incluir sus datos personales para ser informados personalmente de la clasificación del riesgo obtenido. 
Mauricio Ortigosa Hernández

\section{RESULTADOS}

Los 42 profesionales del servicio de urgencias de la Ese Hus, en los respectivos horarios de trabajo, dieron respuestas efectivas de llenado del cuestionario $\mathrm{MBI}$, siendo interesante como el promedio de edad de los mismos oscila en 38 años, la experiencia laboral entre 13 años y en la institución cursan en media aritmética 7 años de antigüedad laboral. De igual forma, del total de los profesionales, los médicos generales representan el $29 \%$, con un $24 \%$ las jefes de enfermería, seguidos de fisioterapeutas con el $21 \%$, médicos especialistas con el $19 \%$ y nutricionista con el $7 \%$. A su vez, el nivel académico de profesional se impone con un $45 \%$, seguido con las especializaciones no médico quirúrgicas con un $26 \%$.
El $40 \%$ de los profesionales son solteros, seguidos por la categoría de $29 \%$ constituidos en unión libre, casados con unos $15 \%$, divorciados con un $11 \%$ y viudas con el $5 \%$. Por otro lado, con el $35 \%$ los profesionales mencionan que hacen turnos matutinos y el $29 \%$ son horarios de trabajo nocturnos (Tabla 1.)

Tabla 1.

Variables Sociodemográficas

\begin{tabular}{lll} 
& MEDIA & DE $^{*}$ \\
\hline Edad (Años) & 38 & 9.7 \\
\hline Experticia laboral (Años) & 13.13 & 8,4 \\
\hline Antigüedad en la institución (Años) & 7.33 & 5,11 \\
\hline & Número & $\%$ \\
\hline Profesiones & & 29 \\
\hline Médico general & 12 & 19 \\
\hline Médicos especialistas & 8 & 24 \\
\hline Jefe Enfermería & 10 & 7 \\
\hline Nutricionista & 3 & 21 \\
\hline Fisioterapeutas & 9 & \\
\hline Nivel académico & & 45 \\
\hline Profesional & 19 & 19 \\
\hline Especialización médico quirúrgica & 8 & 26 \\
\hline Especialización NO médico quirúrgica & 11 & 10 \\
\hline Maestría & 4 & 40 \\
\hline Estado civil & & 15 \\
\hline Soltero & 17 & 29 \\
\hline casado & 7 & 11 \\
\hline Unión libre & 12 & 5 \\
\hline Divorciado & 5 & 19 \\
\hline Viuda & 2 & 35 \\
\hline Turno laboral & & 29 \\
\hline Matutino & 15 & 17 \\
\hline Nocturno & 12 & \\
\hline Jornada completa & 7 & \\
\hline Horas programadas & 8 & \\
\hline$*$ Desvaín Están & \\
\hline
\end{tabular}

*Desviación Estándar

Fuente: Autoría Propia 
En la Tabla 2, se puede apreciar como en la subescala de cansancio emocional, las enfermeras jefes con el $90 \%$ son las profesionales que más cansancio emocional reportan, seguido de los médicos especialistas con el 87,5\%, elm $77 \%$ para los fisioterapeutas, $58 \%$ para médicos generales y el $33 \%$ para nutricionistas. Por otro lado, en la subesacala de Despersonalidad, los médicos especialistas con un $100 \%$ y los nutricionistas con ese mis valor, evidencian el mayor porcentaje de despersonalización, seguidas por las enfermeras jefes con un $90 \%$, los fisioterapeutas con un $89 \%$ y médicos generales con un $67 \%$.
De igual forma, para la subescala de realización personal, los resultados más altos impactan en los médicos especialistas con los $100 \%$, seguidos de las enfermeras jefes con un $80 \%$, fisioterapeutas con el $78 \%$, médicos generales con el $75 \%$ y nutricionistas con el $50 \%$.

Finalmente, evidenciando el comportamiento de los resultados de la aplicación del cuestionario, se aprecia que hubo una prevalencia del síndrome de burnout en los médicos generales con el 80\% (8 profesionales), en los médicos especialistas el $88 \%$ (8 profesionales), jefes de enfermería con el $90 \%$ (9 profesionales), nutricionistas con el 33\% (1 profesional) y fisioterapeutas con el síndrome en un $77 \%$ (7 profesionales), con al menos una de las tres subescalas de medición aterradas.

Tabla 2.

Subescaals de medición cuestionario MBI

Subescala de Cansancio Emocional

\begin{tabular}{|c|c|c|c|c|c|c|}
\hline \multirow[b]{2}{*}{ Profesiones } & \multicolumn{2}{|c|}{ Bajo ( $\leq 19)$} & \multicolumn{2}{|c|}{ Medio ( 19-26) } & \multicolumn{2}{|c|}{ Alto $(\geq 27)$} \\
\hline & $\mathrm{n}$ & $\%$ & $\mathrm{n}$ & $\%$ & $\mathrm{n}$ & $\%$ \\
\hline Médico general & 5 & 42 & 4 & 33 & 3 & 25 \\
\hline Médicos especialistas & 1 & 12,5 & 5 & 62,5 & 2 & 25 \\
\hline Jefe Enfermería & 1 & 10 & 6 & 60 & 3 & 30 \\
\hline Nutricionista & 2 & 67 & 1 & 33 & 0 & 0 \\
\hline Fisioterapeutas & 2 & 23 & 4 & 44 & 3 & 33 \\
\hline \multicolumn{7}{|c|}{ Subescala de Despersonalización } \\
\hline & \multicolumn{2}{|c|}{ Bajo $(\leq 6)$} & \multicolumn{2}{|c|}{ Medio ( 7-10) } & \multicolumn{2}{|c|}{ Alto $(>10)$} \\
\hline Profesiones & $\mathbf{n}$ & $\%$ & $\mathrm{n}$ & $\%$ & $\mathrm{n}$ & $\%$ \\
\hline Médico general & 4 & 33 & 6 & 50 & 2 & 17 \\
\hline Médicos especialistas & 0 & 0 & 5 & 62,5 & 3 & 37,5 \\
\hline Jefe Enfermería & 1 & 10 & 5 & 50 & 4 & 40 \\
\hline Nutricionista & 1 & 33 & 2 & 67 & 0 & 0 \\
\hline Fisioterapeutas & 1 & 11 & 3 & 33 & 5 & 56 \\
\hline \multicolumn{7}{|c|}{ Subescala de Realización Personal } \\
\hline & \multicolumn{2}{|c|}{ Bajo $(<33)$} & \multicolumn{2}{|c|}{ Medio ( 34-39) } & \multicolumn{2}{|c|}{ Alto $(>40)$} \\
\hline Profesiones & $\mathrm{n}$ & $\%$ & $\mathrm{n}$ & $\%$ & $\mathrm{n}$ & $\%$ \\
\hline Médico general & 3 & 25 & 6 & 50 & 3 & 25 \\
\hline Médicos especialistas & 0 & 0 & 7 & 87,5 & 1 & 12,5 \\
\hline Jefe Enfermería & 2 & 20 & 5 & 50 & 3 & 30 \\
\hline Nutricionista & 1 & 50 & 0 & 0 & 1 & 50 \\
\hline $\begin{array}{l}\text { Fisioterapeutas } \\
\text { Fuente: Autoría Propia }\end{array}$ & 2 & 22 & 4 & 45 & 3 & 33 \\
\hline
\end{tabular}




\section{DISCUSIÓN}

El síndrome de burnout viene adquiriendo especial importancia en su aparición, debido a las repercusiones que trae el desarrollo del miso y el impacto a nivel laboral y personal (Macarry, 2013). Los profesionales de salud no se escapan a la aparición y a las secuelas de ámbito ocupacional (Cortés y García, 2012) que puede generar el desarrollo de este síndrome particular.

Existen numerosos estudios, que evidencian la prevalencia del síndrome de burnout en profesionales de salud, siendo uno de ellos llevado a cabo por Novoa y Sánchez (2015), quien hace énfasis que en más del $70 \%$ de los profesionales de un área de trabajo especifica asistencial, pueden llegar a generar episodios o la consecución del síndrome.

Así mismo, autores como Mingote y Santiago, (2013), mencionan que en el personal de enfermería, suelen aparecer los índices más altos del síndrome con un $75 \%$, sobre otras profesiones que comparten servicios profesionales en un área específica de trabajo clínico. Para Borda y Navarro (2010), los profesionales en medicina especializada, en especial los médicos especialistas en medicina interna y cirugía, evidencian porcentajes de aparición del síndrome en un $58 \%$ por encima de otras especialidades médicas (Parraga, 2015)

A su vez, Zaldúa y Lodieu, (2015), menciona que los servicios de atención clínica 0 áreas de atención definidas en el modelo de salud, que más implicación tiene en la aparición del síndrome, son las áreas de urgencias, consulta externa y cirugía general; siendo sustentado por Tous (2014), quien menciona que el área de urgencias en comparación con los demás servicios hospitalarios, representan un orden de aparición del síndrome de burnout por encima del $86 \%$, en comparación con los demás.

\section{CONCLUSIONES}

Teniendo en cuenta el desarrollo del objetivo de investigación, es necesario afirmar que la aparición del síndrome de burnout, es hoy en día a nivel de instituciones de salud, frecuente de aparición y de generar secuelas de orden administrativo y de salubridad que atentan con el buen desarrollo del procesos prestacional de servicios de

salud profesional en el área de trabajo definida de atención. Así mismo, en el resultado de la aplicación del instrumento $\mathrm{MBI}$, se obtiene que en más del $77 \%$ de los profesionales de salud que responden el cuestionario mencionado (excepto en los nutricionistas), evidencia aparición del síndrome de burnout y secuelas de la génesis del mismo, que colocan en riesgo su calidad de salud y una adecuada prestación de servicios profesionales en favor de un usuario consultante y demandante de servicios.

Los profesionales en enfermería jefe, médicos generales y especialistas, representan el mayor porcentaje de aparición (> 80\%), constituyendo un espacio fuerte de intervención, con el fin de mitigar estos factores de aparición; teniendo en cuenta que esta profesiones son los de mayor contacto y presencia en la intervención de un cuadro de salud que demande servicios prestacionales en un paciente.

Por último, es necesario adelantar acciones que permitan mitigar factores de riesgo de aparición del síndrome de burnout en el personal de atención en salud, con el fin de evitar in adecuados esquemas de atención y tratamiento profesional, sumado a incapacidades, ausentismos laborales y demás características que termine en la alteración del buen curso administrativo y de manejo optimo institucional, que a ciencia cierta perjudica de forma clara la atención del servicio y la calidad del mismo. 


\section{BIBLIOGRAFÍA}

Adali E. (2013). Síndrome del Quemado en el personal de enfermería psiquiátrica de hospitales griegos. The European Journal of Psychiatry, 17, 161-170.

Alpi, S. y Alvaran, L. (2014). El Síndrome de Burnout en una Muestra de Auxiliares de Enfermería: Un Estudio Exploratorio. Medellín, Colombia Univ. Psychol. Bogotá (Colombia) 3 (1): 35-45,

Aranda, C. (2010). Factores psicosociales y sindrome de burnout en médicos del primer nivel de atención, en Investigación en Salud, Vol. 8, №. 2

Astudillo, W. y Mendinueta, C. (2010). Syndrome in palliative care. Support Care Center; 4(6):408415.

Barrios, A. y Sánchez, A. (2015). Síndrome de Burnout en Médicos de Hospitales Públicos de la ciudad de Corrientes, en Revista de Posgrado de la Vla Cátedra de Medicina, No. 141, pp. 27-30.

Beas M. (2010). Burnout en mujeres: un estudio comparativo entre contextos de trabajo y no trabajo; congreso "Aspectos psicosociales del estrés ocupacional", Universitat Jaume I.

Borda, M. y Navarro, E. (2010). Síndrome de Burnout en estudiantes de internado del Hospital Universidad del Norte. Salud Uninorte. Barranquilla (col),23(1): 43-51

Chalving, V. (2010). Related stress and occupational Burnout in AIDS caregivers: Test of a coping model with nurses providing. AIDS Care, 12: 149161.

Cortés, A. y Garcia, J. (2012). Estudio comparativo del síndrome de Burnout en profesionales de oncologia: incidencia y gravedad. Medicina Paliativa, $7: 481$

García, G., \& Larios-Gómez, E. (2018). El clima laboral en universidades privadas con enfoque religioso católico y evangélico en Puebla-México. Revista Ide@s Concyteg. 2018(175).

Garrosa, E. y González, J. (2010). Personalidad, burnout y salud. Escritos de Psicología, 4, pp. 64-77.
Guevara, C. y Herrera, A. (2011). Síndrome de desgaste profesional en médicos Hospital Universitario del Valle, Cali, Escuela de Medicina, Facultad de Salud, Universidad del Valle, Cali. Colomb Med; 35:173-178.

Hamson, W. (2015). A social competence model or Burnout. Stress and Burnout in the human services professions. The New Journald England;31: 29-31

Hernández, P. (2012). Estudio descriptivo del síndrome de Burnout en el personal del hospital militar central. Revista Colombiana de Psicología, 7: 71-83.

Jaurequi, M. (2012). El síndrome de Burnout en los profesionales de la salud. Publicación virtual de la Facultad de Psicología y Psicopedagogía de la USAL

Macarry, C. (2013). New Directions In Research And Intervention. Curr Dir Psychol Sci. 12:189-92.

Mingote, R. y Santiago, T. (2013). Estrés en la enfermería, Madrid, España, Díaz de Santos Ediciones.

Misth, U. (2010). Patterns of Burnout among a national sample of public contac workers. Journal of Health and Human Resources Administration, 7 : 189-212.

Moreno, B. (2011). Desgaste profesional (Burnout), personalidad y Salud Percibida. En J. Buendía, F. Ramos (Eds) Empleo, Estrés y Salud. (pp.59 83), Madrid: Pirámide

Novoa, M. y Sánchez, C. (2015). Relación entre Perfil Psicológico, Calidad de Vida y Estrés en Personal de Enfermería. Pontificia Universidad Javeriana. Univ. Psychol. Bogotá (Colombia) 4 (1): 63-75

Parraga, J. (2015). Efectividad del Programa para reducir el Síndrome de Burnout y mejorar las disfunciones emocionales de los profesionales sanitarios. Departamento de Psicología y Sociología de la Educación. Universidad de Extremadura, Cáceres

Peiró, J. (2010). Desencadenantes del estrés laboral. Madrid: Editorial Huddema.

Peñacoba, C. (2010). Estrés asistencial en los servicios de 
salud. En: Simón MB, editor. Manual de Psicología de la salud. Madrid. Casa Editorial Siglo XXI. p.739 - 64 .

Pereira, A; Gálvez, F. y Herrer, M. (2011). Estudios del Burnout: los estudios transculturales BrasilEspaña, en la Revista Colombiana de Psicología, No. 21, pp. $70-88$.

Resolución Número 8430 de 1993 (Octubre 4). Ministerio de salud. República de Colombia

Rivera, D. (2016). Síndrome de Burnout, Aproximaciones Teóricas. Resultado de Algunos Estudios en Popayán. Universidad del Valle.

Romero, R. (2010). Síndrome de desgaste profesional. Formación Médica Continuada, 5, (8), 493-508

Salazar, M. y Beaton, R. (2010). Ecological Model Of Occupational Stress: Application To Urban Firefighters. Aaohn Journal.18;260-8.

Tordera, N. y Mañas, M. (2011). Does roles stress predict Burnout over time among health care professionals? Journal of Psychology and Health, 16: 511-525.

Tous J. (2014). Quemarse en el trabajo. Revista salud pública y nutrición de la Habana, volumen 12 No. 11. Abril-Junio

Vega, N; Sanabria, A. y Sanchez, M. (2012). Síndrome De Desgaste Profesional. Rev Colomb Cir. 2009;24:138-46.

Zaldúa, G. y Lodieu, M. (2015). Dilemas éticos relacionados con el Síndrome de Burnout. Editorial Dunken Bs. As, 23-24 